\title{
Modified Stochastic Petri Net-Based Modeling and Optimization of Emergency Rescue Processes during Coal Mine Accidents
}

\author{
Xiao Li $\mathbb{D}{ }^{1}$ Yongkui Shi $\mathbb{D}^{1},{ }^{1}$ Zaiyong Wang $\mathbb{D}^{1,2}$ and Wenquan Zhang $\mathbb{D}^{1,2}$ \\ ${ }^{1}$ College of Energy and Mining Engineering, Shandong University of Science and Technology, Qingdao 266590, China \\ ${ }^{2}$ State Key Laboratory of Strata Intelligent Control and Green Mining Co-founded by Shandong Province and the Ministry of Science \\ and Technology, Qingdao 266590, China
}

Correspondence should be addressed to Yongkui Shi; shiyongkui@163.com

Received 25 May 2021; Accepted 24 July 2021; Published 14 August 2021

Academic Editor: Dan Ma

Copyright ( $) 2021$ Xiao Li et al. This is an open access article distributed under the Creative Commons Attribution License, which permits unrestricted use, distribution, and reproduction in any medium, provided the original work is properly cited.

\begin{abstract}
The coal mine accidents seriously affect the safety and efficiency of mining for coal mining enterprises. The reliable emergency rescue (ER) processes are explored to minimize the loss of accidents. This paper introduces the stochastic Petri net (SPN) and Markov chain (MC) models based on the system structure flow to analyze the ER processes of coal mine accidents. In addition, a triangle fuzzy strategy is presented to optimize the SPN model. The "9.28" major water inrush accident in Shanxi Fenxi Zhengsheng Coal Company of China is adopted to evaluate the time performance and accident data of the ER process. The MC model-based steady-state probabilities of the system under various states are used to calculate the average delay time of this system. The triangular fuzzy strategy is used to analyze the change value of the total time in the ER system at the unit transition speed when the firing rate of each transition is changed, which finds the most time-consuming key activities in the ER process. The results show that SPN and MC can reflect the dynamic behaviors of ER process, which provides a reference for the rescue operations of other coal mine accidents. The triangular fuzzy strategy can quickly find out the key activities affecting the ER time, which greatly decreases the calculations generated by analyzing the total time of the system changed at the unit transition speed.
\end{abstract}

\section{Introduction}

Coal provides strong supports for the rapid development of the national economy as one of the main energy sources in China. However, frequent safety accidents during coal mining hindered the healthy development of coal mining enterprises. Government and enterprises have tried to change such a passive situation by improving safety management measures and introducing rescue equipment, but the accidents and casualties have not been fundamentally resolved [1-3]. The relevant accident statistics in 2020 show that 122 coal mines accidents occurred in China. Compared with 2019, the death toll caused by coal mine accidents slightly increased. For example, the "1.12" coal dust explosion in Shannxi Baiji Mining Company induced 21 deaths, and the
"12.16" coal gas outburst accident in Guizhou Guanglong coal mine resulted in 16 deaths and 1 injury [4]. Therefore, the establishment of an early warning and rapid rescue framework for coal mine accidents is the key point of current works, which would improve the level of emergency rescue (ER) and reduce or even avoid the casualty caused by accidents $[5,6]$.

To date, several studies have deeply investigated the ER measures of coal mine accidents $[7,8]$. The ER communication system can effectively improve the monitoring accuracy and the efficiency of searching dangerous situations [9]. It is found that the emergency stations covering all mining areas are set up underground to provide medical first aid and transportation of injured in the shortest time [10]. In addition, it is significant for rescue to improve 
the existing refuge chamber and strengthen the escape simulation training [11].

For major coal mine accidents, the core tasks of various ER processes are to construct the mechanism of ER response and strengthen the evaluation of emergency management. It is well known that effective evaluation and optimization of ER processes in coal mines can improve the efficiency of ER and reduce casualties $[12,13]$. A Petri net is an important tool to deal with discrete events, and it is widely used in various fields. Petri nets, as a visual tool for modeling and analysis of the system simulation, can accurately simulate the dynamic behavior of the system and analyze the model characteristics through the definition of assumptions. With the application in various complex systems, Petri nets have evolved into many advanced forms such as color networks, stochastic networks, and object networks. Stochastic Petri nets (SPN) based on time have more advantages in describing the dynamic behavior of a system network. Therefore, SPN is used to study the unreasonable problems in the ER process of the coal mines, which can improve the efficiency of ER and increase the survival probability of trapped miners [14-17].

In this paper, based on the "9.28" major water inrush accident, a coal mine ER model is constructed through SPN, and the effectiveness of the model is analyzed. The steady-state probability, the busy rate of the places, and the utilization rate of transitions are calculated and discussed by MC. The triangular fuzzy strategy is used to analyze the change value of the total time in the ER system after changing the firing rate of each transition and find out the key activities affecting the rescue time.

\section{Related Work}

2.1. Major ER Policies. In 1978, a national meeting about coal mine ER held by the Ministry of Coal Industry of China provided reliable policy supports for the development of coal mine ER. As time goes on, the coal mine ER management system is gradually improved, the ER team is expanded, and the capability of ER is additionally significantly improved. These improvements can be proved by the fact that $34 \%$ of 1363 trapped persons were rescued in 2009, and $72 \%$ of 306 trapped persons were rescued in 2014 [18]. Since there are still some gaps compared with developed countries, the Chinese government attaches great importance to improve the ER management system and has proposed to establish an emergency management department. It is believed that this measure will encourage the further improvement of ER level in coal mines.

2.2. Structure of the ER Team. The rescue team and the medical team are the two main components of the ER team. So far, China has built 482 coal mine rescue teams, which are distributed all over the country. The medical team is divided into the national and provincial rescue centers and mine enterprise rescue stations [19]. The rescue ability of the rescue team is very important, so it is necessary to strengthen the daily traditional physical training and skill training. The application of high-tech in the field of ER enhances the prac- tical ability of rescue teams and improves the efficiency of rescue work such as the development of a comprehensive simulation training system for ER, which achieves personnel positioning and environmental monitoring [20,21].

\subsection{Introduction of ER Equipment and Communication}

Technology. The rescue teams are required to equip with a lot of rescue equipment to deal with different types of coal mine accidents. For instance, the large drainage equipment and mining submersible pumps are used to deal with flood disaster, the rapid sealing and spraying machine are used to deal with fire accidents, the positive pressure blower and exhaust fan are used to deal with gas and coal dust explosion accidents, and the light disaster relief drill is used to deal with roof accidents. Besides, life detectors, support vehicles of multifunctional rescue equipment, and detection robots have also been developed. The use of advanced equipment has greatly enhanced the ability of ER and improved the survival possibility of trapped miners [22-24].

With the development of Internet technology, many new communication technologies have been applied to mine ER such as the video communication between the underground and ground and between the accident scene and ER command departments at all levels $[25,26]$.

\section{Research Methodology}

At first, SPN was used to describe and analyze the causal relationship between computer system events. After more than a century of development and improvement, SPN has been extensively used in other fields such as finite state machines, communication protocol, synchronous control, production system, and business process. SPN has the characteristics of graphic visualization and mathematical logic, which can describe highly discrete complex systems [27-31].

3.1. Definition of SPN. SPN can simulate and analyze discrete events of the system during an uncertain time. SPN is a seven-tuple directed graph including place, transition, arc, and firing rate [32].

$$
\mathrm{SPN}=(P, T, F, K, W, M, \Lambda),
$$

(1) $N=(P, T, F)$ refers to a basic network

(2) $K: P \longrightarrow N^{+} \cup\{\omega\}, K$ is the capacity of $P$ on SPN, $k=\infty$

(3) $W: F \longrightarrow N^{+}, W$ is a weight function of $F$ on $N$

(4) $M: P \longrightarrow N$ meet a condition $\forall p \in P, M(p) \leq K(p)$, $M_{0}$ is the state marking of SPN

(5) $\Lambda: T \longrightarrow R^{+}$represents firing function, which is quantitatively analyzed by the firing rate

3.2. Basic Relationship. Events $t_{i}, t_{j} \in T$ have a sequential relationship on mark $M$. That is, $M\left[t_{i}\right\rangle M^{\prime} \wedge-M\left[t_{j}\right\rangle \wedge M^{\prime}\left[t_{j}\right\rangle$ [33], as shown in Figure 1. 


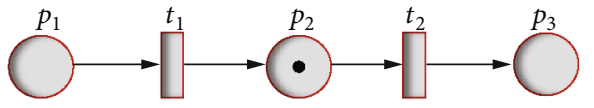

FIGURE 1: Sequential relationship of an SPN model.

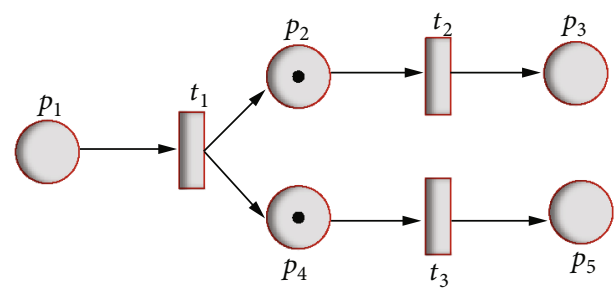

FIGURE 2: Concurrency relationship of an SPN model.

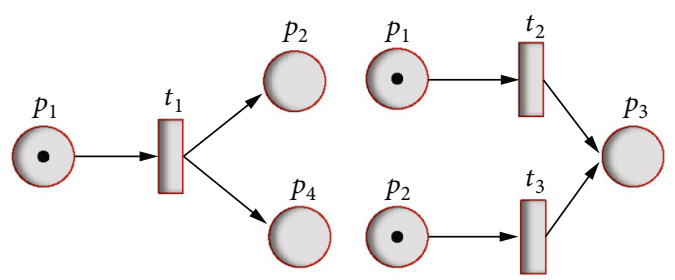

Figure 3: Conflicted relationship of an SPN model.

Events $t_{i}, t_{j} \in T$ accept the weight on mark $M$, resulting in a concurrency relationship, the satisfied relations are as follows:

$$
\begin{gathered}
M\left[\left\{t_{i}, t_{j}\right\}\right\rangle \Leftrightarrow \forall p \in P . W\left(p, t_{i}\right)+W\left(p, t_{j}\right) \leq M(p) \leq K(p) \\
-W\left(t_{i}, p\right)-W\left(t_{j}, p\right),
\end{gathered}
$$

$$
\begin{aligned}
M\left[\left\{t_{i}, t_{j}\right\}\right\rangle M^{\prime} \Leftrightarrow & {\left[\left\{t_{i}, t_{j}\right\}\right\rangle \wedge \forall p \in P . M^{\prime}(p)=M(p)+W\left(t_{i}, p\right) } \\
& +W\left(t_{j}, p\right)-W\left(p, t_{i}\right)-W\left(p, t_{j}\right),
\end{aligned}
$$

as shown in Figure 2.

Events $t_{i}, t_{j} \in T$ have a conflicted relationship on mark $M$ [33]. That is, $t_{i} \neq t_{j} \wedge M\left[t_{j}\right\rangle \wedge \neg M\left[\left\{t_{i}, t_{j}\right\}\right\rangle$, as shown in Figure 3 .

3.3. Model Construction of SPN. The SPN model is shown in Figure 4. $\left\{p_{1}, p_{2}, p_{3}, p_{4}, p_{5}\right\}$ refers to a set of classical places. $\left\{t_{2}, t_{2}, t_{3}\right\}$ refers to a transition set containing time. For example, $t_{1}$ represents the time taken from $p_{1}$ to $p_{2}$ and $p_{3}$. After $t_{1}$ is fired, $p_{2}$ generates two tokens and $p_{3}$ generates one token. The number marked on the directed arc indicates the weight. If there is no number, the weight is 1 .

The analysis process of the SPN model is as follows: firstly, give place $p_{1}$ a token to fire transition $t_{1}$, so that place $p_{2}$ generates two tokens and place $p_{3}$ generates one token. Then, it is judged whether the number of tokens in places $p_{2}$ and $p_{3}$ meets the condition of firing transition $t_{2}$, and if so, place $p_{4}$ can be reached. Finally, we judge the condition of firing transition $t_{3}$ according to the previous method. If the condition is met, it will reach the termination place $p_{5}$, and the process will end.
The implementation steps of using the SPN model to analyze the performance of the ER process for coal mine accidents are as follows [34-36]:

(1) The systematic SPN model is established to verify the validity of the model. Here, $T$-invariant method is chosen to realize this process. A linear algebraic matrix equation is constructed to determine the performance of the SPN model

$$
C y=(O-I) y=0,
$$

where $C$ refers to the incidence matrix, $O$ refers to the postmatrix, $I$ is the prematrix, and $Y$ is a row vector, representing $T$-invariant

(2) According to the initial mark $M_{0}$ and firing rate $\lambda$, the isomorphic Markov chain (MC) is obtained [37]

(3) The MC is studied. Based on the steady-state probability, the system performance is analyzed and evaluated. The steady-state probability in MC is regarded as a row vector $X=\left(x_{1}, x_{2}, \cdots, x_{n}\right)$, and the linear equations $X Q=0$ are listed

$$
\begin{aligned}
X Q & =0, \\
\sum x_{i} & =1,1 \leq i \leq n,
\end{aligned}
$$

where $Q$ represents the state transition matrix

The linear equations are calculated to obtain the steadystate probability.

$$
\begin{aligned}
P\left[M_{i}\right] & =x_{i}(1 \leq i \leq n), \\
Q & =\left[q_{i j}\right] .
\end{aligned}
$$

For the nondiagonal element $q_{i j}$, $q_{i j}=\left\{\begin{array}{l}q_{i j}, \text { when the state } M_{i} \text { can reach the state } M_{j}, \text { the line is marked } q_{i j}, \\ 0, \text { when the states } M_{i} \text { can not reach the state } M_{j} .\end{array}\right.$

For the diagonal element $q_{i j}$,

$$
q_{i j}=-\Sigma_{k} q_{i j}
$$

where $k$ refers to the number of states connected to $M_{i}$.

$\forall p \in P, \forall i \in N, P[M(p)=i]$ is the probability that each $p$ with $i$ marks. The busy probability of place indicator can be calculated using Equation (8).

$$
P[M(p)=i]=\Sigma p\left[M_{j}\right]
$$




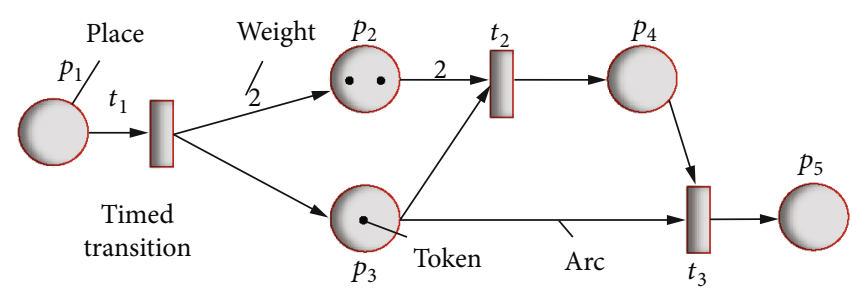

FIgURE 4: Representation of the SPN model.

The utilization of a transition indicator refers to the sum of all steady-state probability of firing transition $t$ and is calculated as follows:

$$
U(t)=\Sigma_{m \in E} P[M] .
$$

The token rate of transition indicator represents the average token number of transition flowing into the place $p$ of transition $t$ per unit time. The calculation formula is as follows:

$$
R(t, p)=w(t, p) \times U(t) \times \lambda .
$$

Suppose $\forall p \in P$, the average token number $\bar{N}$ of the system refers to the average value of marks contained by $p_{i}$ in any reachable marking graph. The marked average value of the set $p_{i} \in P$ of a place is the sum of the average value of each place.

$$
\bar{N}=\Sigma j \times P\left[M\left(p_{i}\right)=j\right] .
$$

The average delay time $t$ of the system is the ratio of the average token number $\bar{N}$ and the token rate $R(t, p)$ of transition.

$$
\bar{T}=\frac{\bar{N}}{R(t, p)} .
$$

3.4. The Optimization Strategy Based on Triangular Fuzzy (TF). The TF is a widely used function of fuzzy mathematics, which is mainly suitable for the situation of insufficient field data and low precision of monitoring instruments. The variables are fuzzified to form a trust interval with the upper and lower limits $[38,39]$. Assume that the fuzzy variable is

$$
\tilde{E}=\left(e^{-}, e, e^{+}\right), e^{-} \leq e \leq e^{+},
$$

where $e$ refers to the value of the variable. $e^{-}$and $e^{+}$represent the lower and upper limits of fuzzy variables, respectively.
The corresponding triangular membership function is

$$
\eta_{E}= \begin{cases}0, & x<e^{-}, \\ \frac{x-e^{-}}{e-e^{-}}, & e-\leq x \leq e, \\ \frac{e^{+}-x}{e^{+}-e}, & e \leq x \leq e^{+}, \\ 0, & x>e^{+},\end{cases}
$$

where $\eta_{E}$ is the membership degree of the fuzzy variable and $x$ is a variable.

The triangular membership function is calculated by using $X$ cut set to obtain a fuzzy interval.

$$
\begin{aligned}
E^{(a)} & =\left\{\min \left[e^{(a)}\right], \max \left[e^{(a)}\right]\right\} \\
& =\left[e^{-}+\left(e-e^{-}\right) a, e^{+}-\left(e^{+}-e\right) a\right] \quad a \in[0,1] .
\end{aligned}
$$

Optimization steps of the triangular fuzzy are as follows:

(1) The firing rate $\tilde{\lambda}=\left(\lambda_{1}, \lambda_{2}, \cdots, \lambda_{n}\right)$ in the SPN model is fuzzified according to different proportions [40]. The fuzzy intervals $\left[\lambda_{i}^{-}, \lambda_{i}\right]$ and $\left[\lambda_{i}, \lambda_{i}^{+}\right]$of firing rate $\lambda_{i}$ are constructed

(2) $a$ is valued in the interval $[0,1]$, which meets the rule of increasing step by step. For example, $a=0,0.1$, $0.2, \cdots, 1$. Substituting different $a$ values into formula (15) to obtain $E_{i}^{(a)}$. Then, substituting $\lambda^{\prime}=\left(\lambda_{1}, \lambda_{2}\right.$, $\left.\cdots \lambda_{i-1}, E_{i}^{a}, \lambda_{i+1}, \cdots, \lambda_{n}\right)$ into the SPN mode to calculate the interval $\left[\min _{0 \leq a \leq 1}\left(\bar{T}_{i}^{a}\right), \max _{0 \leq a \leq 1}\left(\bar{T}_{i}^{a}\right)\right](i=1,2, \cdots, n)$

(3) The total time of system change under unit transition rate is

$$
\delta_{i}=\left|\frac{\max \left(T_{i}^{a}\right)-\min \left(T_{i}^{a}\right)}{\lambda_{i}^{+}-\lambda_{i}^{-}}\right|
$$

(4) When $\delta_{i}$ is larger than most $\delta$ values, it shows that the change of transition has great influences on the average delay time of the system. Therefore, the optimized SPN model can be obtained only by simulating $\delta_{i}$ with a large numerical value 


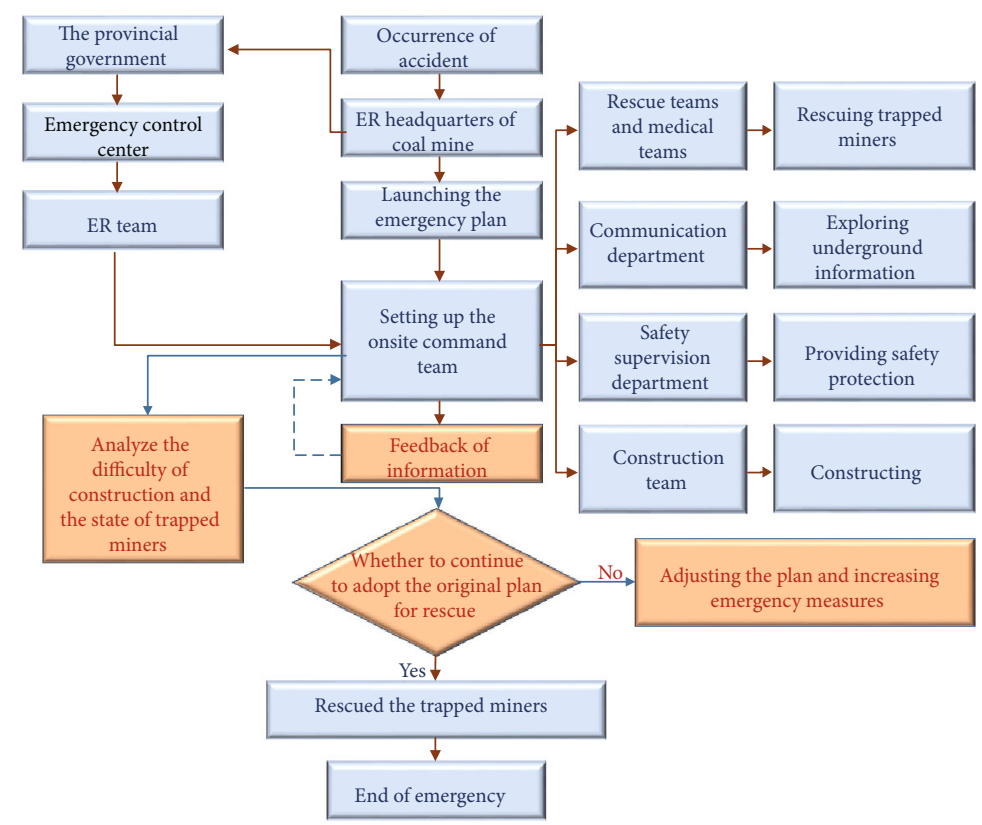

FIgURE 5: The ER process of the coal mine.

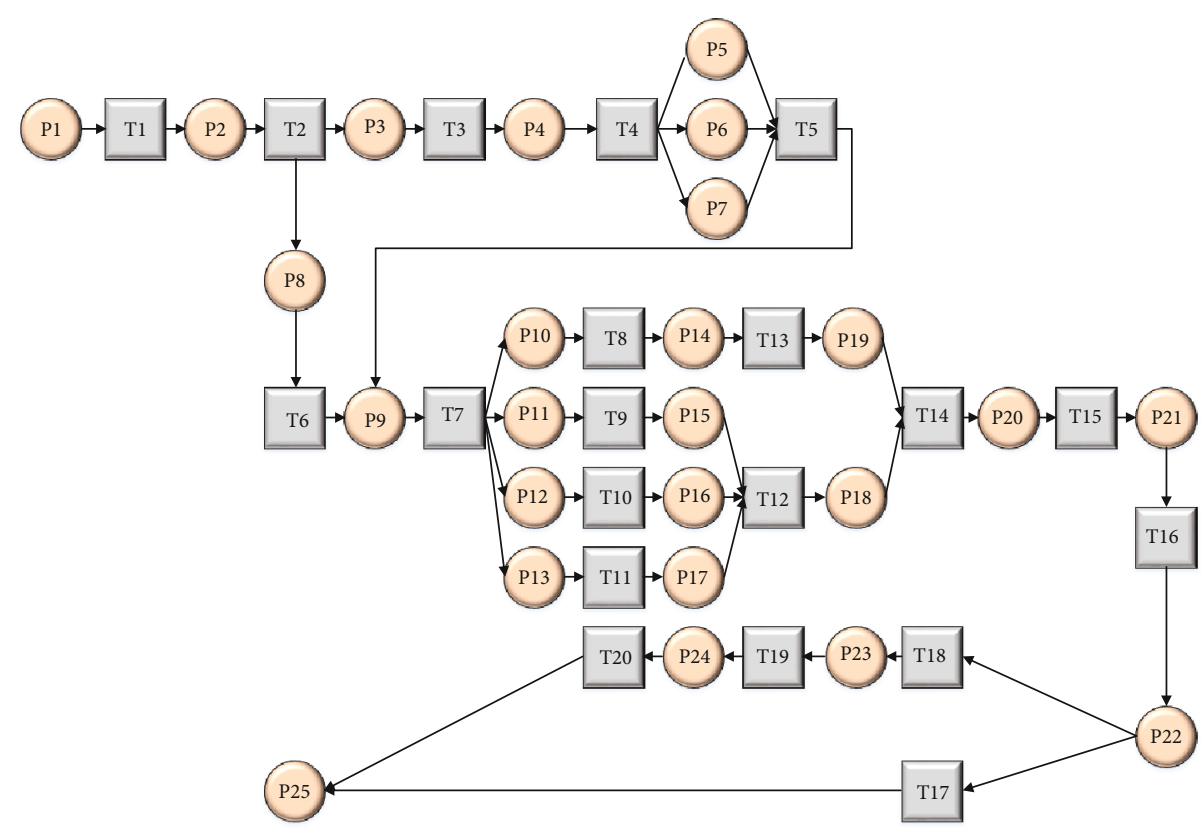

FIgURE 6: The SPN of ER process during coal mine accident.

\section{Case Study}

4.1. Case Introduction. The "9.28" major water inrush accident in the Shanxi Fenxi Zhengsheng Coal Company of China is used as a case to validate the proposed model. In this case, the water inrush occurred in the east flank return airflow roadway to the coal mine dispatching room. Though the leader of underground miners executed emergency evacuations immediately, many miners were still trapped for the far distance between the working face and the shaft. According to the guidance of higher authorities, coal mine leaders executed ER plan, set up an ER headquarter, and launched rescue operations. The ER process for this accident is shown in Figure 5.

\subsection{Performance Analysis of Water Inrush ER Process Based} on SPN Model. Figure 6 shows the SPN model of the ER process. The SPN model combines all the ER activities and points out the relationship among these activities, as shown in Figure 6.

In Figure 6, a place represents the event state and a transition represents the event activity during the ER process. The process includes 25 places and 20 transitions, and their respective definitions are shown in Table 1. 
TABLE 1: Definitions of places and transitions.

\begin{tabular}{|c|c|c|c|}
\hline & Places & & Transitions \\
\hline P1 & Accident occurrence & $\mathrm{T} 1$ & Reflecting accident information \\
\hline $\mathrm{P} 2$ & ER headquarters of coal mine & $\mathrm{T} 2$ & Reporting the accident to the superior \\
\hline P3 & The provincial government & T3 & Issuing rescue command \\
\hline $\mathrm{P} 4$ & Emergency control center & $\mathrm{T} 4$ & Scheduling according to the authority \\
\hline P5 & Experts & T5 & Going to the coal mine \\
\hline P6 & Rescue teams and medical teams & T6 & Analyzing emergency conditions and making ER plan \\
\hline P7 & Technicians & T7 & Allocating resources and organizing team \\
\hline P8 & Implement emergency plan & T8 & Starting preparatory work by the communication department \\
\hline P9 & Set up the onsite command team & T9 & $\begin{array}{l}\text { Starting preparatory work by the rescue teams and medical } \\
\text { teams }\end{array}$ \\
\hline P10 & Communication department & $\mathrm{T} 10$ & Starting preparatory work by the safety supervision department \\
\hline P11 & Rescue teams and medical teams & $\mathrm{T} 11$ & Starting preparatory work by the construction team \\
\hline P12 & Safety supervision department & $\mathrm{T} 12$ & Draining in flooded roadway \\
\hline P13 & Construction team & $\mathrm{T} 13$ & Exploring information underground \\
\hline P14 & Communications department that finished preparatory work & $\mathrm{T} 14$ & Finding the location of the trapped miners \\
\hline P15 & $\begin{array}{l}\text { The rescue teams and medical teams that finished preparatory } \\
\text { work }\end{array}$ & $\mathrm{T} 15$ & Reporting to the onsite command team \\
\hline P16 & $\begin{array}{l}\text { The safety supervision department that finished preparatory } \\
\text { work }\end{array}$ & T16 & Analyzing the difficulty of construction and the state of victims \\
\hline P17 & Construction team that finished preparatory work & $\mathrm{T} 17$ & Rescuing the trapped miners \\
\hline P18 & Advance of rescue roadway & T18 & Adjusting emergency plan and increasing emergency measures \\
\hline P19 & General information & T19 & Adding large rescue equipment to join in rescue \\
\hline P20 & Important information & $\mathrm{T} 20$ & $\begin{array}{l}\text { Providing first aid to the victims by rescue teams and medical } \\
\text { teams }\end{array}$ \\
\hline $\mathrm{P} 21$ & Information received by the command team & & \\
\hline P22 & Judgment of accident grade & & \\
\hline P23 & Greater rescue efforts & & \\
\hline P24 & Key rescue sections that have been breakthrough & & \\
\hline P25 & End of ER & & \\
\hline
\end{tabular}

$T$-invariant and $P$-invariant are common methods to analyze the validity of the model. The validity of the model is analyzed by the $T$-invariant method [41]: $Y_{1}=(1,1,1,1$, $1,1,1,1,1,0,0,1,0,1,1,1,1,1,1,1,1)$ and $Y_{2}=(1,1,1,1,1$, $1,1,1,0,1,1,1,1,1,1,1,1,1,1,1,1)$.

$Y_{1}$ and $Y_{2}$ have nonnegative integer solutions. The existence of $T$-invariant in the SPN model means that the SPN model has the properties of reachability and liveness. In addition, $Y_{1}>0$ and $Y_{2}>0$ mean that the SPN model is bounded which proves that the SPN model is correct and reasonable [42].

\section{Results and Discussion}

The time parameters that obeyed a certain probability distribution are added in the SPN, and the time of random variables is associated with the transitions. Through the accident investigation, the time consumed in each link of the accident is collected to obtain the average time $t$ of each transition. According to the firing rate formula, the average firing rate of 20 transitions is obtained, and the results are as shown: $\lambda=1 / \mathrm{t}=(1 / 10,1 / 5.1 / 5,1 / 20,1 / 150,1 / 20 / 1 / 60,1 /$
$15,1 / 15,1 / 15,1 / 20,1 / 170,1 / 720,1 / 120,1 / 5,1 / 20,1 / 200,1 / 10$, $1 / 200,1 / 150)$.

5.1. Isomorphic MC. The performances of the SPN and MC models are consistent, and a bounded SPN is equivalent to a finite MC. The structure and performance analysis of the SPN model are integrated into the MC model to obtain the isomorphic MC, which can better analyze the SPN performance. The flow chart of the MC is shown in Figure 7.

According to formulas (4), (8), and (9), the steady-state probability, the busy rate of the places, and the utilization rate of the transitions are calculated, respectively, as shown in Tables 2-4.

In Table 3, the busy rates of places of P9 and P14 P19 are higher, which indicates that the state information of these places cannot be effectively processed and results in the accumulation of information.

The onsite command team (place P9) is composed of coal mine leaders, experts, technicians, rescue team, and medical team, which formulates and adjusts the ER plan according to the onsite situation, and maintains communication with relevant superior departments. Since the experts of research 


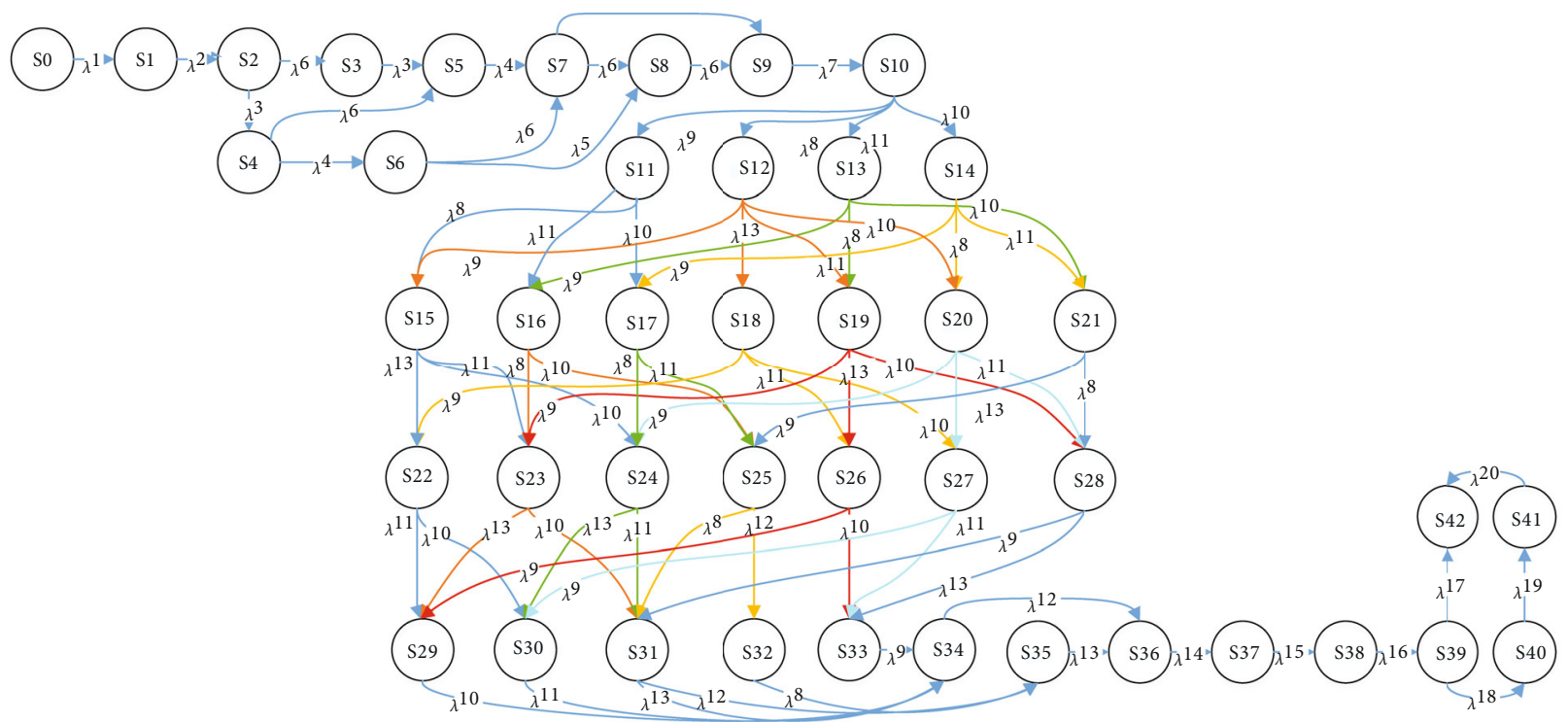

FIgURE 7: Isomorphic MC equivalents to the SPN model.

TABLE 2: Steady-state probability.

\begin{tabular}{lccccc}
\hline $\begin{array}{l}\text { Steady- } \\
\text { state } \\
\text { probability }\end{array}$ & Value & $\begin{array}{c}\text { Steady- } \\
\text { state } \\
\text { probability }\end{array}$ & Value & $\begin{array}{c}\text { Steady- } \\
\text { state } \\
\text { probability }\end{array}$ & Value \\
\hline$P\left(M_{0}\right)$ & 0.00634 & $P\left(M_{14}\right)$ & 0.00092 & $P\left(M_{28}\right)$ & 0.00205 \\
$P\left(M_{1}\right)$ & 0.00190 & $P\left(M_{15}\right)$ & 0.00104 & $P\left(M_{29}\right)$ & 0.00006 \\
$P\left(M_{2}\right)$ & 0.00254 & $P\left(M_{16}\right)$ & 0.00066 & $P\left(M_{30}\right)$ & 0.00016 \\
$P\left(M_{3}\right)$ & 0.00063 & $P\left(M_{17}\right)$ & 0.00105 & $P\left(M_{31}\right)$ & 0.22132 \\
$P\left(M_{4}\right)$ & 0.00507 & $P\left(M_{18}\right)$ & 0.00001 & $P\left(M_{32}\right)$ & 0.00004 \\
$P\left(M_{5}\right)$ & 0.00761 & $P\left(M_{19}\right)$ & 0.00065 & $P\left(M_{33}\right)$ & 0.00006 \\
$P\left(M_{6}\right)$ & 0.00448 & $P\left(M_{20}\right)$ & 0.00104 & $P\left(M_{34}\right)$ & 0.23313 \\
$P\left(M_{7}\right)$ & 0.09063 & $P\left(M_{21}\right)$ & 0.00066 & $P\left(M_{35}\right)$ & 0.22339 \\
$P\left(M_{8}\right)$ & 0.00060 & $P\left(M_{22}\right)$ & 0.00002 & $P\left(M_{36}\right)$ & 0.00634 \\
$P\left(M_{9}\right)$ & 0.03804 & $P\left(M_{23}\right)$ & 0.00205 & $P\left(M_{37}\right)$ & 0.00317 \\
$P\left(M_{10}\right)$ & 0.00254 & $P\left(M_{24}\right)$ & 0.00406 & $P\left(M_{38}\right)$ & 0.00634 \\
$P\left(M_{11}\right)$ & 0.00092 & $P\left(M_{25}\right)$ & 0.00207 & $P\left(M_{39}\right)$ & 0.00576 \\
$P\left(M_{12}\right)$ & 0.00092 & $P\left(M_{26}\right)$ & 0.00001 & $P\left(M_{40}\right)$ & 0.03458 \\
$P\left(M_{13}\right)$ & 0.00063 & $P\left(M_{27}\right)$ & 0.00002 & $P\left(M_{41}\right)$ & 0.08646 \\
\hline
\end{tabular}

institutes and universities were in different cities, they cannot arrive at the accident site in time, which caused the waste of the ER time. Therefore, the emergency center should improve work efficiency and optimize deployment time.

After the accident, the busy rate of the places of the P14 and P16 (coal enterprise) is very high. Through deep analysis, it is found that the number of emergency training is less and the rescue work is not proficient enough for employees in the communication department and the safety supervision department. The mine leaders should pay attention to the daily emergency training and strengthen the emergency response awareness of the miners.
TABLE 3: The busy rate of the places.

\begin{tabular}{lccc}
\hline Place & Value & Place & Value \\
\hline$P\left[M\left(p_{1}\right)=1\right]$ & 0.00634 & $P\left[M\left(p_{13}\right)=1\right]$ & 0.01268 \\
$P\left[M\left(p_{2}\right)=1\right]$ & 0.00190 & $P\left[M\left(p_{14}\right)=1\right]$ & 0.45652 \\
$P\left[M\left(p_{3}\right)=1\right]$ & 0.00317 & $P\left[M\left(p_{15}\right)=1\right]$ & 0.46655 \\
$P\left[M\left(p_{4}\right)=1\right]$ & 0.01268 & $P\left[M\left(p_{16}\right)=1\right]$ & 0.46655 \\
$P\left[M\left(p_{5}\right)=1\right]$ & 0.09511 & $P\left[M\left(p_{17}\right)=1\right]$ & 0.46338 \\
$P\left[M\left(p_{6}\right)=1\right]$ & 0.09511 & $P\left[M\left(p_{18}\right)=1\right]$ & 0.22978 \\
$P\left[M\left(p_{7}\right)=1\right]$ & 0.09511 & $P\left[M\left(p_{19}\right)=1\right]$ & 0.23980 \\
$P\left[M\left(p_{8}\right)=1\right]$ & 0.01268 & $P\left[M\left(p_{20}\right)=1\right]$ & 0.00317 \\
$P\left[M\left(p_{9}\right)=1\right]$ & 0.17556 & $P\left[M\left(p_{21}\right)=1\right]$ & 0.00634 \\
$P\left[M\left(p_{10}\right)=1\right]$ & 0.00951 & $P\left[M\left(p_{22}\right)=1\right]$ & 0.00576 \\
$P\left[M\left(p_{11}\right)=1\right]$ & 0.00951 & $P\left[M\left(p_{23}\right)=1\right]$ & 0.03458 \\
$P\left[M\left(p_{12}\right)=1\right]$ & 0.00951 & $P\left[M\left(p_{24}\right)=1\right]$ & 0.08646 \\
\hline
\end{tabular}

TABLE 4: Utilization rate of transitions.

\begin{tabular}{lccc}
\hline Transition & Value & Transition & Value \\
\hline$U\left(t_{1}\right)$ & 0.00634 & $U\left(t_{11}\right)$ & 0.01270 \\
$U\left(t_{2}\right)$ & 0.00190 & $U\left(t_{12}\right)$ & 0.45652 \\
$U\left(t_{3}\right)$ & 0.00317 & $U\left(t_{13}\right)$ & 0.45652 \\
$U\left(t_{4}\right)$ & 0.01268 & $U\left(t_{14}\right)$ & 0.00634 \\
$U\left(t_{5}\right)$ & 0.09511 & $U\left(t_{15}\right)$ & 0.00317 \\
$U\left(t_{6}\right)$ & 0.01269 & $U\left(t_{16}\right)$ & 0.00634 \\
$U\left(t_{7}\right)$ & 0.03804 & $U\left(t_{17}\right)$ & 0.00576 \\
$U\left(t_{8}\right)$ & 0.00565 & $U\left(t_{18}\right)$ & 0.00576 \\
$U\left(t_{9}\right)$ & 0.00951 & $U\left(t_{19}\right)$ & 0.03458 \\
$U\left(t_{10}\right)$ & 0.00951 & $U\left(t_{20}\right)$ & 0.08646 \\
\hline
\end{tabular}


The medical rescue team (place P15) includes medical rescue stations of the mining enterprise and medical personnel assisted by other areas. Because the two teams had not trained together before, the cooperation of rescue teams was not good enough, which caused the redundancy of place P15.

The construction team (place P17) mainly includes the rescue team from the provincial mine rescue base and the mine enterprise rescue team. Because many rescue machines are required to be transported, it is very likely that vehicles will be congested and cannot arrive quickly due to the influence of traffic conditions.

Places P18 and P19 are in a synchronized state. Due to the uncertainty of the accident location and the influence of the complex underground environment on the equipment such as life detectors, it is difficult to find the location of the trapped miners for a short time. In the early stage of the rescue, the rescue team can only use drainage equipment to drain the floodwater in the roadway. Therefore, we should increase the number of rescue equipment and use more advanced rescue equipment to deal with ER of coal mine accidents.

It can be seen from Table 4 that the utilization rates of transitions T12 and T13 are much higher than that of the remaining transitions, which indicates that the activities they represented spend more time.

T12 indicates the draining in the flooded roadway. During this process, it is difficult to determine the exact position of the trapped miners, so the ER team can only gradually rescue along the rescue roadway based on the known information and experience. In the flooded roadway, life detectors and other equipment are used to detect the vital signs nearby. This work is difficult because it not only needs to clean up the accumulated water and gravel in the roadway but also needs to stabilize the roadway to ensure its safety, which leads to the total time of T12 is very long.

T13 indicates the exploring information underground. In this process, because the communication staff need to follow the construction team to carry out underground detection and feedback the real-time information to the ER headquarters immediately, which also takes a lot of time.

The average delay time of the system can be calculated according to formulas (10)-(12): $\bar{T}=\bar{N} / R(t, p)=2.7702$ / $0.0101=274.44 \mathrm{~min}$.

5.2. Optimization Analysis of ER Process. In this process, the SPN model of the emergency rescue process was analyzed, the busy places and high utilization of transitions were identified, and improvement measures were suggested. We changed some transition's time and calculated the corresponding average delay time of the whole ER system.

The method of changing the firing rate of each transition is adopted to optimize the total delay time of the system. When the speed range of the firing rate of each transition is constant, the different decreases of the total delay time have different impacts on the whole ER system. A lot of computations are needed to substitute the firing rate of each transition into the SPN model, so a triangular fuzzy strategy is proposed to optimize the SPN, which greatly simplifies the calculation.
TABLE 5: Changed value of the total time of the ER system when the transition unit changed.

\begin{tabular}{lccccc}
\hline Transition & Unit value & Sort & Transition & Unit value & Sort \\
\hline$\lambda_{12}$ & 45889.04 & 1 & $\lambda_{14}$ & 224.91 & 11 \\
$\lambda_{13}$ & 44605.57 & 2 & $\lambda_{10}$ & 209.04 & 12 \\
$\lambda_{19}$ & 2702.56 & 3 & $\lambda_{9}$ & 209.04 & 13 \\
$\lambda_{20}$ & 915.48 & 4 & $\lambda_{17}$ & 155.05 & 14 \\
$\lambda_{5}$ & 907.76 & 5 & $\lambda_{18}$ & 134.36 & 15 \\
$\lambda_{7}$ & 460.77 & 6 & $\lambda_{1}$ & 128.00 & 16 \\
$\lambda_{6}$ & 287.16 & 7 & $\lambda_{3}$ & 71.50 & 17 \\
$\lambda_{11}$ & 265.23 & 8 & $\lambda_{15}$ & 71.27 & 18 \\
$\lambda_{4}$ & 238.93 & 9 & $\lambda_{2}$ & 71.00 & 19 \\
$\lambda_{16}$ & 235.59 & 10 & $\lambda_{8}$ & 36.26 & 20 \\
\hline
\end{tabular}

Firstly, 5\% fuzzy degree is set for each transition to get the fuzzy interval of each transition according to formula (15). Then, the average delay time of the system corresponding to the transition is calculated in the SPN model. Finally, according to formula (16), the change value $\delta$ of the total time of the ER system is calculated when each transition unit changes, and the results are shown in Table 5.

Table 5 shows that when the fuzzy degrees of transitions are same, the $\delta$ values corresponding to transitions are quite different. The value of $\delta_{12}$ corresponding to $\lambda_{12}$ is 1265 times of $\delta_{8}$ corresponding to $\lambda_{8}$. Therefore, changing the value of $\lambda_{12}$ can reduce the total system time more than changing the value of $\lambda_{8}$. In addition, the values of $\lambda_{13}, \lambda_{19}, \lambda_{20}$, and $\lambda_{5}$ have the same effect on reducing the total time, so only five parameters need to be changed to optimize the SPN model.

Firstly, it is assumed that only the value of $\lambda_{12}$ is changed and the remaining values are constant. The results are shown in Figure 8.

Figure 8 shows that when the value of $\lambda_{12}$ changes from 0 to 0.003 , the probability of $P\left(M_{34}\right)=(\mathrm{P} 15, \mathrm{P} 16, \mathrm{P} 17, \mathrm{P} 19)$ is greatly decreased. The probability of $P\left(M_{31}\right)=(\mathrm{P} 14, \mathrm{P} 15, \mathrm{P}$ $16, \mathrm{P} 17)$ is slightly increased when the value of $\lambda_{12}$ changes from 0 to 0.0009 but decrease gradually when the value of $\lambda_{12}$ changes from 0.0009 to 0.003 .

With the advancing speed of the rescue roadway in the mining area increases, the communication department will find the location of trapped miners underground faster in the rescue process and promptly report the rescue progress to the ER headquarters of coal mine. The command team masters the latest rescue situation in order to quickly adjust the plan and deploy rescue measures. Therefore, it is necessary to carry out a reasonable allocation of emergency resources and special drainage equipment so that the medical rescue team, the safety supervision department, the construction team, and the communication department can quickly put into the ER work. Meanwhile, the safety supervision department, communication department, and medical rescue team from the mine enterprise must strengthen the daily management and emergency training. The emergency center should request nearby hospitals to allocate medical teams for 


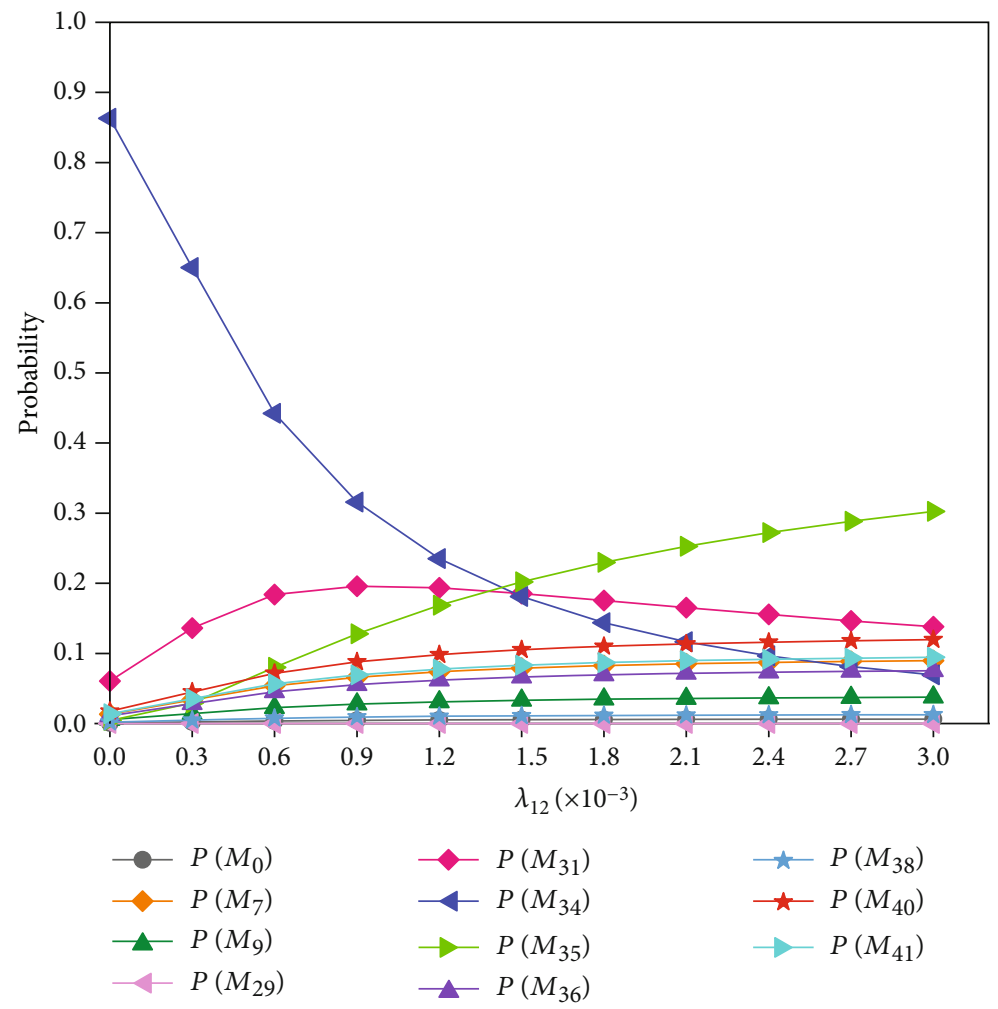

Figure 8: Variation of system probability with change in $\lambda_{12}$.

support, but it is necessary to ensure that these rescue teams are well trained.

Secondly, it is assumed that only the value of $\lambda_{13}$ is changed and the remaining values are constant. The results are shown in Figure 9.

Figure 9 shows that when the value of $\lambda_{13}$ changes from 0 to 0.003 , the probability of $P\left(M_{35}\right)=(\mathrm{P} 14, \mathrm{P} 18)$ is greatly decreased. The probability of $P\left(M_{31}\right)=(\mathrm{P} 14, \mathrm{P} 15, \mathrm{P} 16, \mathrm{P} 17$ ) is slightly increased when the value of $\lambda_{13}$ changes from 0 to 0.0009 , but gradually decreased when the value of $\lambda_{13}$ changes from 0.0009 to 0.003 .

The probability value and variation range of $P\left(M_{31}\right)$ in Figure 10 is basically consistent with that in Figure 9. This result indicates that if the location information of trapped miners can be grasped as soon as possible, the rescue work of the communications department, rescue teams and medical teams, safety supervision department, and construction team will proceed successfully. With the increase of $\lambda_{13}$, the probability value of $P\left(M_{35}\right)$ gradually decreases from 0.86151 to 0.06308 . It means that the communications department works more efficiently, and the rescue roadway advances faster and strives for golden rescue time for trapped miners. Therefore, more detection equipment is needed to expand the range of underground information exploration, to grasp the location information of trapped miners and feedback information to the headquarters faster.

Thirdly, it is assumed that only the value of $\lambda_{19}$ is changed and the remaining values are constant. The results are shown in Figure 10.
Figure 10 shows that when the value of $\lambda_{19}$ changes from 0 to 0.01 , the probability of $P\left(M_{40}\right)=(\mathrm{P} 23)$ is greatly decreased. The rescue team encountered obstacles in the process of roadway advancement. To solve this problem, the command team immediately adjusted the plan to increase emergency rescue efforts. The most effective way is to mobilize large rescue equipment to remove obstacles in the roadway, which will shorten the rescue time. Therefore, we need to ensure the supply of large-scale rescue equipment to deal with some serious problems in water inrush accidents. The "expanding ER" should also be added to the emergency plan to improve the efficiency of ER.

Fourthly, it is assumed that only the value of $\lambda_{20}$ is changed and the remaining values are constant. The results are shown in Figure 11.

Figure 11 shows that when the value of $\lambda_{20}$ changes from 0 to 0.01 , the probability of $P\left(M_{41}\right)=(\mathrm{P} 24)$ is greatly decreased. It can be explained that when the rescue speed of the trapped miners is accelerated, the distance between the rescuer and the trapped person is decreased. In this way, if there are some miners in poor health, the rescuer can transport the drugs to the trapped miners by drilling, so that they can get emergency treatment and prevent the deterioration of illness. For this reason, the medical team should prepare all kinds of first-aid medicines in advance to deal with the possible illness of the miners.

Finally, it is assumed that only the value of $\lambda_{5}$ is changed and the remaining values are constant. The results are shown in Figure 12. 


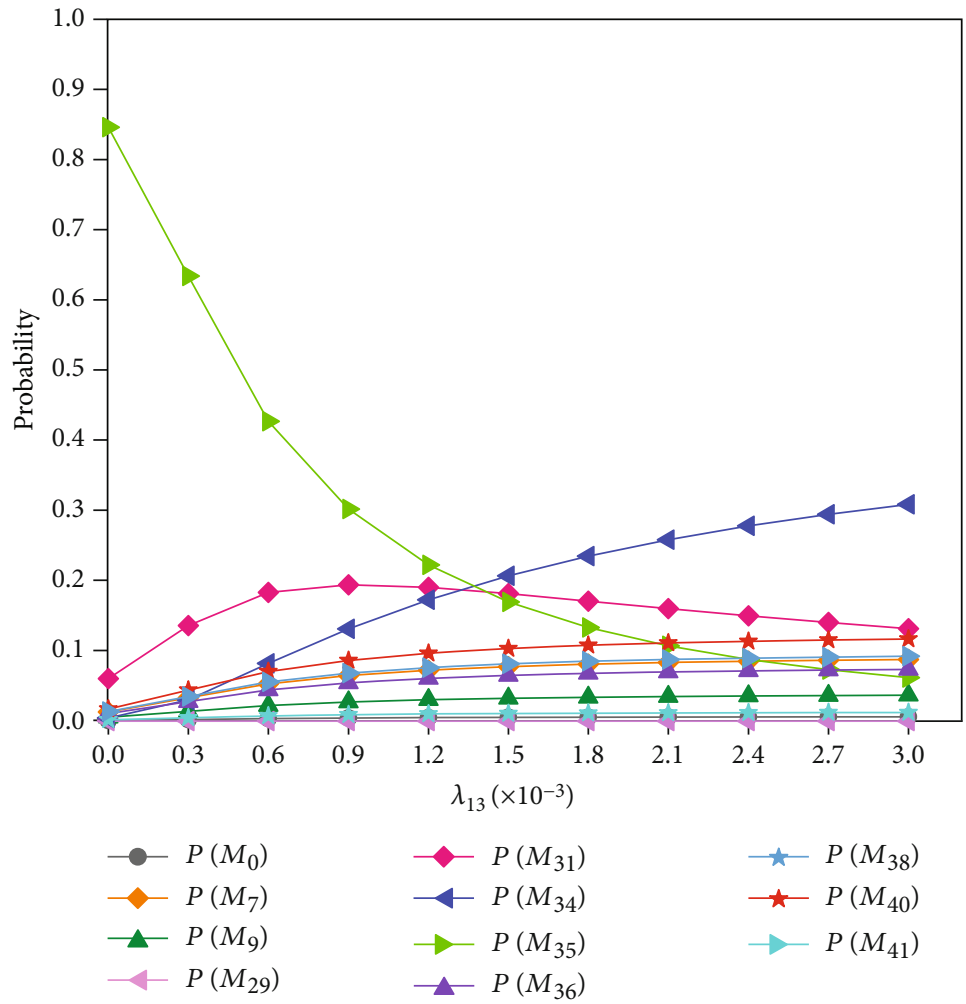

Figure 9: Variation of system probability with change in $\lambda_{13}$.

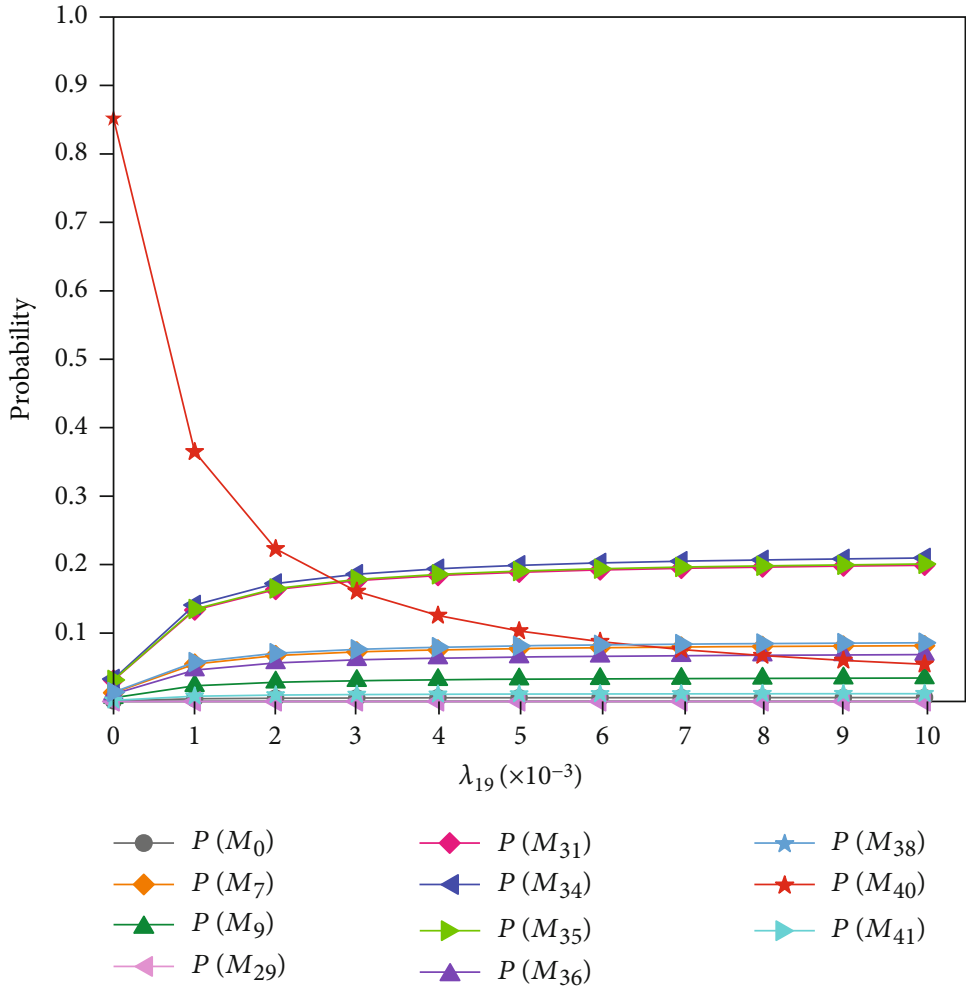

FIgURE 10: Variation of system probability with change in $\lambda_{19}$. 


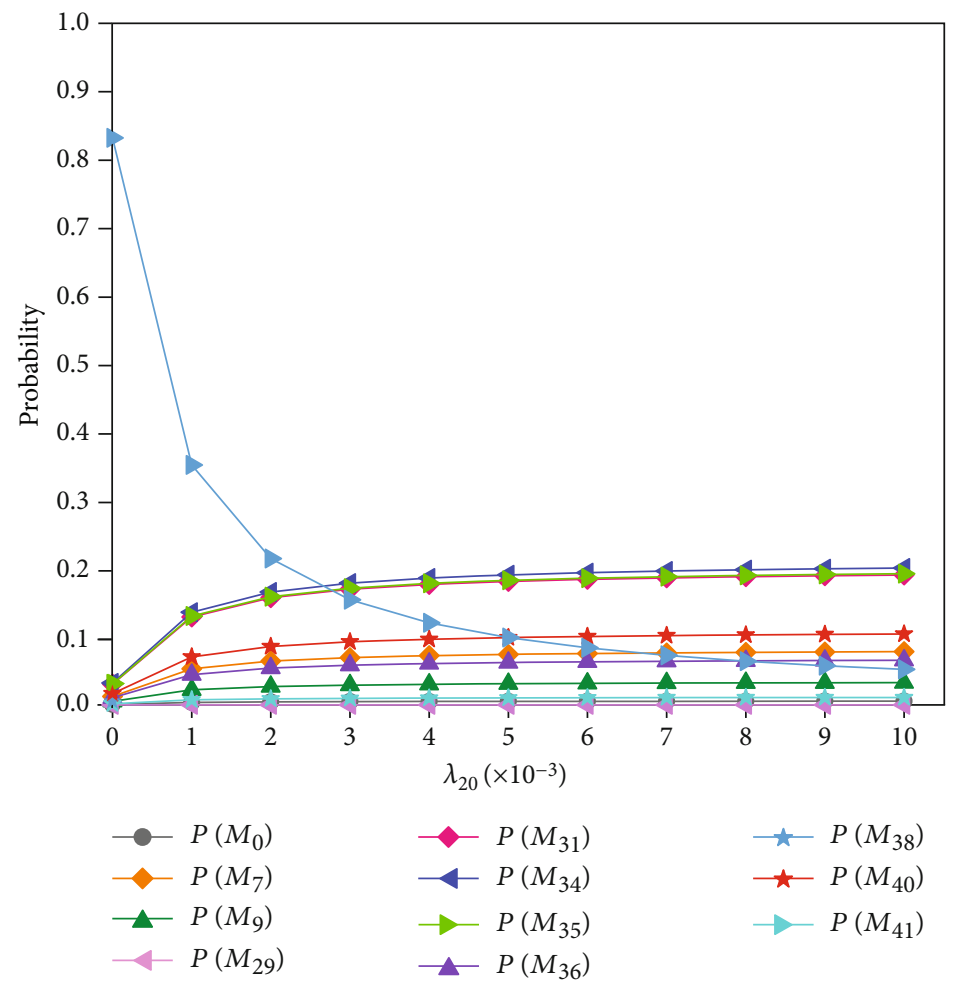

FIGURE 11: Variation of system probability with change in $\lambda_{20}$.

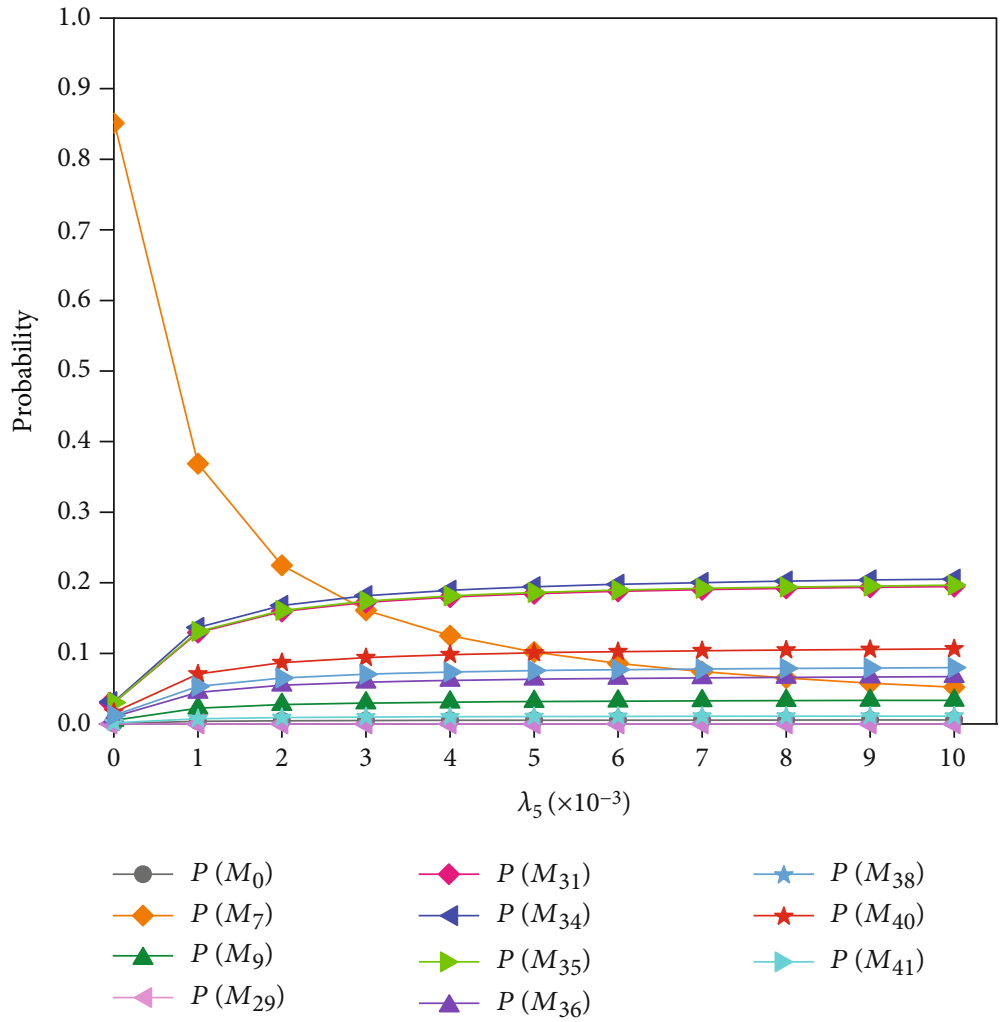

FIGURE 12: Variation of system probability with change in $\lambda_{5}$. 
Figure 12 shows that when the value of $\lambda_{5}$ changes from 0 to 0.01 , the probability of $P\left(M_{7}\right)=(\mathrm{P} 5, \mathrm{P} 6, \mathrm{P} 7, \mathrm{P} 9)$ is greatly decreased. It can be explained that if the speeds of the experts, rescue teams, medical teams, and technicians arrive at the accident site are larger, the on-site command team can be set up more quickly. However, because most of the experts are distributed in universities and research institutes, it takes the longest time for them to arrive at the coal mine, which has a great influence on the establishment of the onsite command team. Therefore, the government should provide an emergency green channel for experts to ensure that they can arrive at the accident site faster.

Above all, in the process of ER, coal mining enterprises should be equipped with large rescue equipment to deal with emergencies. When multicooperation is carried out for ER, it is necessary to optimize resource allocation, coordinate internal and external relations, enhance the awareness of cooperation, and organize daily emergency training, which will be better to carry out ER operations.

\section{Conclusions and Further Work}

6.1. Conclusions. In this paper, a new method is proposed to model and simulate the coal mine ER process based on modified SPN. To validate the correctness of this method, the SPN method is applied to the major water inrush accident in Shanxi Fenxi Zhengsheng Coal Company.

(1) The theoretical method of SPN is adopted to establish the ER process of coal mine and found that busy rates of places P9 and P14 P19 are higher and the utilization rates of translations T12 and T13 are higher. In addition, the problems existing in these places and transitions are analyzed

(2) The triangular fuzzy strategy was introduced into the SPN. The change value of the total time of the ER system is analyzed when each transition changes, which illustrates that the key activities affecting the rescue time

(3) In order to better carry out the ER process, it is suggested that larger rescue equipment should be added, team training should be organized regularly, cooperation should be strengthened, and green channels should be provided for experts

\subsection{Further Work}

(1) The ER of the coal mine is a very complex process, which can divide many links in more detail, such as organizational structure and emergency supplies. Through this division, a more complete ER process and SPN model can be constructed

(2) When the SPN model is analyzed, it is assumed that the SPN model obeys an exponential distribution. However, this assumption may have some differences with the actual conditions. Therefore, more in-depth researches should be conducted to help us choose a more appropriate probability distribution
(3) Visualization software should be developed for coal mines, which can show the ER process more vividly and give more supports on the ER work using information technology

\section{Data Availability}

All data, models, or code generated or used during the study are available from the corresponding author by request.

\section{Conflicts of Interest}

The authors declare no conflicts of interest.

\section{Acknowledgments}

This work was supported by the National Natural Science Foundation of China (51774199) and the Natural Science Foundation of Shandong Province (ZR2017BEE001).

\section{References}

[1] X. S. Zhang, S. S. Li, and X. H. Zhang, "Evaluation of emergency rescue ability based on RS-IPA: evidence from coal mining firms," Natural Hazards, vol. 106, no. 3, pp. 1915-1929, 2021.

[2] Z. Wang, Q. Zhang, J. Shao, W. Zhang, X. Wu, and X. Zhu, "New type of similar material for simulating the processes of water inrush from roof bed separation," ACS Omega, vol. 5, no. 47, pp. 30405-30415, 2020.

[3] G. Fu, X. Xie, Q. Jia, W. Tong, and Y. Ge, “Accidents analysis and prevention of coal and gas outburst: understanding human errors in accidents," Process Safety and Environmental Protection, vol. 134, pp. 1-23, 2020.

[4] T. F. Xu and X. B. Wang, "Statistics and regularity analysis of gas explosion accidents in domestic low-gas coal mines in recent ten years," Mining safety \& Environmental Protection, vol. 48, no. 3, pp. 126-130, 2021.

[5] G. Zhang, W. Zhang, H. Wang, S. Cao, Y. Wu, and Z. Wang, "Research on arch model and numerical simulation of critical water and sand inrush in coal mine near unconsolidated layers," Geofluids, vol. 2020, 12 pages, 2020.

[6] Q. Wu, Z. Du, Y. Zhao, H. Xu, and X. Zhang, "Optimal location of water level sensors for monitoring mine water inrush based on the set covering model," Scientific reports, vol. 11, no. 1, 2021.

[7] A. E. Halim and J. F. Brune, "Do refuge chambers represent a good strategy to manage emergencies in underground coal mines?" Mining Metallurgy \& Exploration, vol. 36, no. 6, pp. 1191-1199, 2019.

[8] Z. F. Hu, "Research on case representation and retrieval of coal mine accidents," in 2013 International Conference on Computational and Information Sciences, pp. 462-465, 2013.

[9] X. Li, Y. K. Shi, and C. M. Pang, "Evaluation model of coal mine emergency rescue resource allocation based on weight optimization TOPSIS method," in IOP Conference Series: Earth and Environmental Science, vol. 651, 2021no. 3.

[10] J. Wang, B. Hu, L. Dong, J. Chang, and W.-P. Wang, "Safety pre-control of stope roof fall accidents using combined event tree and fuzzy numbers in China's underground noncoal mines," IEEE Access, vol. 8, pp. 177615-177622, 2020.

[11] T. Phenrat, "Community citizen science for risk management of a spontaneously combusting coal-mine waste heap in Ban 
Chaung, Dawei District, Myanmar," GeoHealth, vol. 4, no. 6, p. e2020GH000249, 2020.

[12] Y. Liu, Z. Fan, and H. Qi, "Dynamic statistical evaluation of safety emergency management in coal enterprises based on neural network algorithms," Journal of Intelligent \& Fuzzy Systems, vol. 39, no. 4, pp. 5521-5534, 2020.

[13] Z. He, Q. Wu, L. Wen, and G. Fu, "A process mining approach to improve emergency rescue processes of fatal gas explosion accidents in Chinese coal mines," Safety Science, vol. 111, pp. 154-166, 2018.

[14] Y. Zhang, Y. Xie, Y. Zhang, J. Qiu, and S. Wu, "The adoption of deep neural network (DNN) to the prediction of soil liquefaction based on shear wave velocity," Bulletin of Engineering Geology and the Environment, vol. 80, no. 6, pp. 5053-5060, 2021.

[15] L. Liu, W. Liu, and Y. Y. Du, "Business process modeling and analysis based on queue logical Petri nets," International Journal of Software Engineering and Knowledge Engineering, vol. 29, no. 1, pp. 115-137, 2019.

[16] J. I. Latorre-Biel, D. Ferone, A. A. Juan, and J. Faulin, "Combining simheuristics with Petri nets for solving the stochastic vehicle routing problem with correlated demands," Expert Systems with Applications, vol. 168, 2020.

[17] T. Elusakin, M. Shafiee, T. Adedipe, and F. Dinmohammadi, "A stochastic Petri net model for O\&M planning of floating offshore wind turbines," Energies, vol. 14, no. 4, p. 1134, 2021.

[18] J. Xi, D. Cliff, and Z. Wu, "A comparison of underground coal mine emergency management in China and Australia," International Journal of Emergency Management, vol. 13, no. 4, p. 349, 2017.

[19] L. L. Li, Study on the Secondary Explosion Rule in Disaster Area and Emergency Decision-Making Model of Coal Mine Gas Explosion, China University of Mining \& Technology-Beijing, 2019.

[20] L. Li, D. Guo, Y. Wang, K. Wang, and R. Lian, “Anatomy of mine rescue teams' casualty incidents: a basis for medical emergency preparedness and injury prevention," Disaster Medicine and Public Health Preparedness, vol. 13, no. 4, pp. 695-699, 2019.

[21] D. Olave-Rojas and S. Nickel, "Modeling a pre-hospital emergency medical service using hybrid simulation and a machine learning approach," Simulation Modelling Practice and Theory, vol. 109, p. 102302, 2021.

[22] Y. Xu, Q. Chang, X. Yan, W. Han, B. Chang, and J. Bai, “Analysis and lessons of a mine water inrush accident resulted from the closed mines," Arabian Journal of Geosciences, vol. 13, no. $14,2020$.

[23] X. Zhao, Q. Wang, B. Xie, S. Pan, and M. Ji, “A dynamic rescue route planning method based on 3D network in mine water inrush hazard," Geomatics, Natural Hazards and Risk, vol. 10, no. 1, pp. 2387-2407, 2019.

[24] S. N. Nazaretyan and S. S. Nazaretyan, "Assessment of the need for rescue forces during destructive earthquakes (a case study of Armenia)," Seismic Instruments, vol. 47, no. 4, pp. 26-42, 2021.

[25] W. Zhang, Z. Wang, X. Zhu, W. Li, B. Gao, and H. Yu, “A risk assessment of a water-sand inrush during coal mining under a loose aquifer based on a factor analysis and the fisher model," Journal of Hydrologic Engineering, vol. 25, no. 8, p. 04020033 , 2020.

[26] M. Onifade, "Towards an emergency preparedness for selfrescue from underground coal mines," Process Safety and Environmental Protection, vol. 149, pp. 946-957, 2021.
[27] Q. Zhang and Z. Y. Wang, Spatial Prediction of Loose Aquifer Water Abundance Mapping Based on a Hybrid Statistical Learning Approach, Earth Science Informatics, 2021.

[28] X.-Y. Li, Y. Liu, Y.-H. Lin, L.-H. Xiao, E. Zio, and R. Kang, “A generalized Petri net-based modeling framework for service reliability evaluation and management of cloud data centers," Reliability Engineering \& System Safety, vol. 207, p. 107381, 2021.

[29] R. M. Cao, L. N. Hao, and Q. Gao, "Modeling and decisionmaking methods for a class of cyber-physical systems based on modified hybrid stochastic timed Petri net," IEEE Systems Journal, vol. 14, no. 4, pp. 4684-4693, 2020.

[30] L. Shi, S. Du, Y. Miao, and S. Lan, "Modeling and performance analysis of satellite network moving target defense system with Petri nets," Remote Sensing, vol. 13, no. 7, p. 1262, 2021.

[31] Q. Yu, L. Cai, and X. Tan, “Airport emergency rescue model establishment and performance analysis using colored Petri nets and CPN tools," International Journal of Aerospace Engineering, vol. 2018, 8 pages, 2018.

[32] M. K. Molloy, "Performance analysis using stochastic Petri nets," IEEE Transactions on Computers, vol. C-31, no. 9, pp. 913-917, 1982.

[33] C. W. Kang, M. Imran, M. Omair, W. Ahmed, M. Ullah, and B. Sarkar, "Stochastic-Petri net modeling and optimization for outdoor patients in building sustainable healthcare system considering staff absenteeism," Mathematics, vol. 7, no. 6, p. 499, 2019.

[34] S. Zairi, B. Zouari, H. Rahal, and J. Pradat-Peyre, "A generic generalized stochastic Petri nets model for the performance analysis of FMS considering the resources failures," in 2018 5th International Conference on Control, Decision and Information Technologies, pp. 737-742, 2018.

[35] A. Finkel and J. Leroux, "Recent and simple algorithms for Petri nets," Software \& Systems Modeling, vol. 14, no. 2, pp. 719-725, 2015.

[36] J. Hao and W. Pei-an, "An approach for workflow performance evaluation based on discrete stochastic Petri net," in IEEE International Conference on e-Business Engineering (ICEBE'07), pp. 327-330, 2007.

[37] X. M. Dong, "A model of airport security work flow based on petri net," IOP Conference Series: Materials Science and Engineering, vol. 242, no. 1, p. 012105, 2017.

[38] X. Li, X. Wang, G. Lang, and H. Yi, "Conflict analysis based on three-way decision for triangular fuzzy information systems," International Journal of Approximate Reasoning, vol. 132, pp. 88-106, 2020.

[39] J. Y. Dong, S. P. Wan, and S. M. Chen, "Fuzzy best-worst method based on triangular fuzzy numbers for multi-criteria decision-making," Information Sciences, vol. 547, pp. 1080 1104, 2021.

[40] F. Tüysüz and C. Kahraman, "Modeling a flexible manufacturing cell using stochastic Petri nets with fuzzy parameters," Expert Systems with Applications, vol. 37, no. 5, pp. 39103920, 2009.

[41] H. Sun, J. Liu, Z. Han, and J. Jiang, "Stochastic Petri net based modeling of emergency medical rescue processes during earthquakes," Journal of Systems Science \& Complexity, vol. 34, no. 3, pp. 1063-1086, 2021.

[42] K. W. G. de Araújo, M. O. de Andrade, R. M. F. Lima, and C. A. L. de Oliveira, "Performance analysis of metropolitan bus rapid transit line via generalized stochastic Petri nets," Journal of Urban Planning and Development, vol. 146, no. 1, 2020. 